\title{
Flexible Low Profile Frequency Selective Surface for X-Band Shielding Applications
}

\author{
Wai Yan Yong ${ }^{1}$, Tom Hartman ${ }^{1}$, Andrés Alayón Glazunov ${ }^{1,2}$ \\ ${ }^{1}$ University of Twente, Department of Electrical Engineering, Enschede, Netherlands \\ ${ }^{2}$ Chalmers University of Technology, Department of Electrical Engineering, Gothenburg, Sweden. \\ w.y.yongwaiyan@utwente.nl
}

\begin{abstract}
This paper considers the design and evaluation of the performance of a low profile frequency selective surface (FSS) with flexible characteristics for $\mathrm{X}$-band shielding applications. The proposed FSS unit cell is realized using a miniaturized ring loop (MRL) which is a transformed version of the conventional ring loop. The evaluation of the performance of the MRL for both planar and conformal configurations is performed using the Computer Simulation Technology (CST) software. First, the unit cell modelling is used to evaluate the performance of the planar MRL at various angles of incidence for both TE and TM polarizations. Second, the semi-infinite modelling technique is employed to evaluate the performance of the FSS in a curved configuration. Simulation results show that the proposed MRL was miniaturized to have a unit cell of $0.25 \lambda_{0}$, where $\lambda_{0}$ is the free-space wavelength at the center frequency. The designed MRL demonstrates a stable transition response up to a $60^{\circ}$ angle of incidence. In addition, it is worthwhile to note that when the proposed FSS is bent, the resonant frequency is slightly shifted by roughly $6 \%$.
\end{abstract}

Index Terms - Frequency Selective Surface (FSS), EMI, shielding, flexible

\section{INTRODUCTION}

Over the past few years, the investigation of the impact of the electromagnetic interference (EMI) on human health and electronic devices has received an extensive attention from both industry and the academic research alike [1]-[4]. The EMI from wireless communication systems may constitute a significant hazard to the operation of medical equipment and military devices [5], [6]. With the advent of (5G) communication systems, a large number of devices are expected to be connected wirelessly with each other as a consequence of the anticipated "big bang" of the Internet of things (IoTs) [5]. In order to mitigate the impact of unwanted signals on the various equipments, a number of solutions have been produced such as the employment of metallic (or reinforced) walls or signal jammers. However, these techniques are impractical in many cases or very costly to put into practice. In order to overcome the ineffectiveness of the above-mentioned approaches, the use of a frequency selective surface (FSS) is an alternative technique for the screening of the undesirable electromagnetic signals [7]. FSSs can also be used as a sub-reflector [8] and in beam-sweeping antenna systems [9].

Recently, there has been a nurturing interest to produce bendable electronic devices [10]. In the wireless communication industry bendable antennas and portable RF devices are of most interest [11]. Even though a number of manufacturing techniques have been proposed to develop flexible FSSs [6], [12], to the best authors' knowledge, only a few previous studies have reported the evaluation of bending effects on the performance of pliable FSS [7], [8].

In this paper, we propose the employment of the semiinfinite modeling technique for the examination of the performance of a new design of a conformal band-stop miniaturized ring loop (MRL) FSS when it is mounted on a curved structure. In order to ensure that the proposed MRL FSS operates well when mounted on a planar as well as on a curved structure, the FSS is first modeled and evaluated with the help of the conventional unit cell modeling technique. This is done to assure that the MRL manages to operate on the planar structure for both the TE and the TM polarizations at normal and oblique angles of incidence up to $60^{\circ}$. The optimized MRL is then modeled as a semi-infinite curved structure for the examination of the bending impact on its performance. Simulation results show that the proposed FSS can be employed as an EMI shield for planar and curved structures.

1

\section{Design Of The FSS Unit Cell}

The miniaturization of the conventional ring loop is realized by adding four slots and embedding four cross-dipoles at each of the $90^{\circ}$ angles of the conventional ring loop FSS as illustrated in Fig. 1. This approach has a detrimental effect on the package density of the ring loop element, the package density is $50 \%$ of a conventional ring loop FSS that usually resonates at $0.5 \lambda_{o}$, where $\lambda_{0}$ is the free-space wavelength of the center frequency. By having a compact unit cell dimension, the frequency response performance of the FSS array will improve, as it reduces the sensitivity of the FSS array towards a wide angle of incidence [7]. The proposed design is computed by using silver nanoparticle ink which is supported by a polyethylene terephthalate (PET) substrate with a thickness $h=0.125 \mathrm{~mm}$, a dielectric constant of 2.7 and a tangent loss of 0.004 . For the silver nanoparticle ink used in the computation, the electrical resistance is defined as $0.3 \Omega / \mathrm{m}$ \footnotetext{
2019

${ }^{1}$ This article has been accepted for publication in EMC Sapporo APEMC
} 


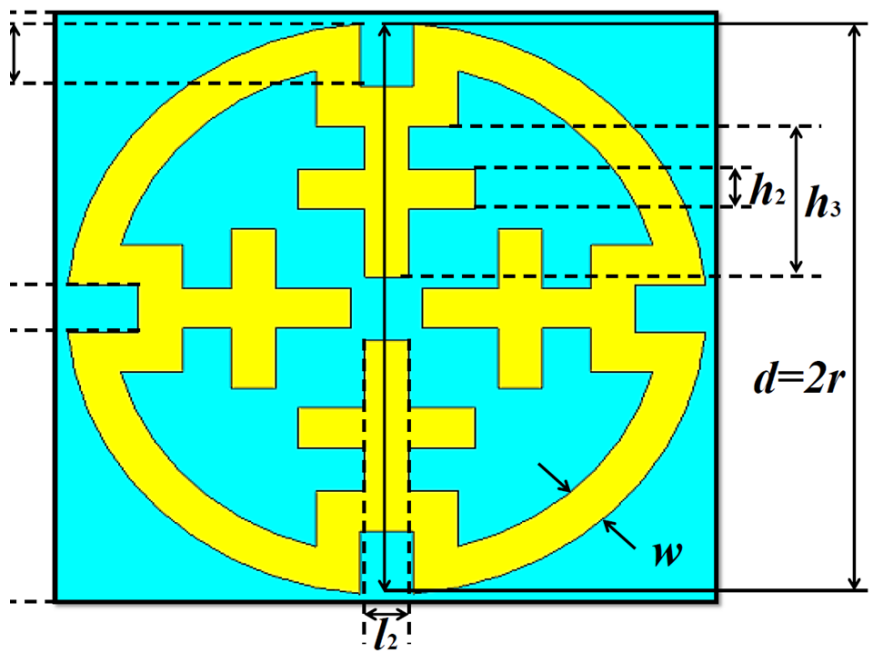

Fig. 1: Miniaturized Ring Loop Element, $p=7.5 \mathrm{~mm}, d=$ $7.2 \mathrm{~mm}, w=0.5 \mathrm{~mm}, l_{1}=0.8 \mathrm{~mm}, l_{2}=0.6 \mathrm{~mm}, h_{1}=$ $0.6 \mathrm{~mm}, h_{2}=0.5 \mathrm{~mm}, h_{3}=1.9 \mathrm{~mm}$

with a thickness of $t_{A g}=0.003 \mathrm{~mm}$, which is commercially supplied by AgIC [13].

\section{Results AND Discussion}

\section{A. Evaluation of the Performance of the Planar FSS}

The unit cell of the proposed MRL FSS is simulated using the CST Microwave Studio software. The simulation is performed using the frequency domain solver as it is suitable for the simulation of a highly resonant structure and it is capable of examining the performance of the FSS at different angles of incidence for both the TE and the TM polarizations. The periodic boundary condition is set in both horizontal and vertical directions and the $\mathrm{z}$-direction is set to be open (add space) for locating the ports. To reduce the simulation load, the CST full floquet port is utilized to simulate the unit cell of the FSS to model the proposed FSS as an infinite array. The simulated transmission response of the proposed FSS at normal incidence of both TE and TM polarizations is shown in Fig. 2. As can be seen from Fig. 2, at the normal incidence the MRL provides a signal attenuation of up to $32 \mathrm{~dB}$. The proposed FSS provides a $-10 \mathrm{~dB}$ bandwidth of around $19 \%$ which is equivalent to $1.9 \mathrm{GHz}$, ranging from $9.1 \mathrm{GHz}$ to 11 GHz. Furthermore, when the angle of incidence increases up to $60^{\circ}$, for both the TE and TM polarizations, the frequency response of the FSS is similar to the normal angle of incidence. Therefore, it can be concluded that the MRL is insensitive to the variation of the angle of incident and polarization.

\section{B. Evaluation of the performance of the Conformal FSS}

In order to scrutinize the performance of the FSS in curved structures, the aforementioned method (i.e., the Floquet port simulation) is not directly applicable for curved FSSs as it is impractical to rearrange the FSS elements in a purely 2D periodic geometry when the FSS is bent. Hence, the unit

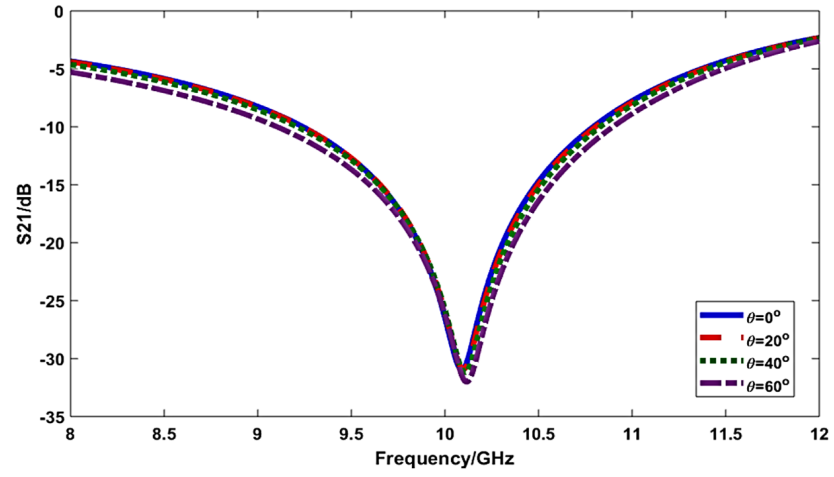

(a)

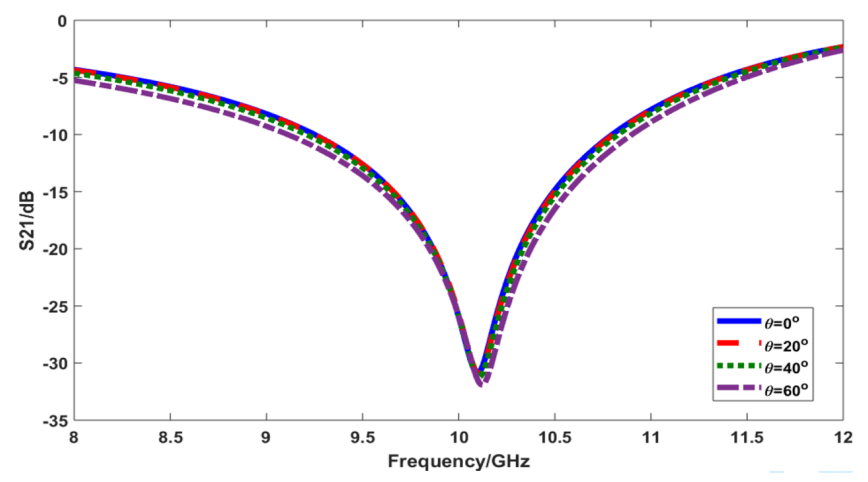

(b)

Fig. 2: Simulated transmission response of the MRL FSS with planar feature at (a) TE polarization and (b) TM polarization at normal and oblique angle of incidence up to $60^{\circ}$

cell simulation in CST is not applicable as it will assume that each and every single unit cell is bent in the same direction. Therefore, in order to overcome the aforementioned deficiency of the unit cell modelling, in this paper, a semi-infinite model is proposed in the evaluation of the bending of the FSS array.

Fig. 3 shows the simulation set-up in CST that is utilized for the examination of the performance of the conformal FSS array. The simulation of the conformal FSS array is completed by drawing a finite single row of the FSS array formed on a semicircle with the help of PEC-PMC boundary conditions. In this case, the bending direction (x-direction) is set with PMC, the periodic direction (y-direction) is set as PEC condition and the z-direction is set with open (add-space). The use of the PEC-PMC boundary condition allows the port exciting a plane wave as PEC boundary force there is no tangential electric field and PMC boundary force there is no tangential magnetic field. By using this simulation set-up, the FSS is assumed infinite along the cylinder axis and finite/terminated on the bend surface. Employing this simulation set-up significantly reduces the computational burden in the evaluation of the conformal FSS array as compared to the simulation of the full finite FSS structure. However, this technique only allows the investigation of the bending performance at normal incidence.

Fig. 4 illustrates the comparison of the simulated 
TABLE I: Performance comparison among the existing flexible FSS

\begin{tabular}{|c|c|c|c|c|}
\hline Ref. & Center Freq. (GHz) & Unit Cell dimension & Realization Technique & Bending Effect \\
\hline$[6]$ & 1.8 & $0.5 \lambda_{o} \times 0.5 \lambda_{o}$ & flexible PET substrate & Not being evaluated \\
\hline$[7]$ & 10 & $0.25 \lambda_{o} \times 0.25 \lambda_{o}$ & flexible PET substrate & $7.89 \%$ Bandwidth reduction \\
\hline$[8]$ & 5 & $0.1 \lambda_{o} \times 0.1 \lambda_{o}$ & flexible polymer substrate & Multiple ripple result in bandwidth enhancement \\
\hline$[14]$ & 5 & $0.07 \lambda_{o} \times 0.07 \lambda_{o}$ & flexible polymer substrate & Multiple ripple result in bandwidth enhancement \\
\hline This Work & 10 & $0.25 \lambda_{o} \times 0.25 \lambda_{o}$ & flexible PET substrate & $3.2 \%$ Bandwidth reduction \\
\hline
\end{tabular}

Notes: $\lambda_{o}$ is the free-space wavelength of the center frequency

transmission response of the planar FSS and the conformal FSS at the normal angle of incidence for TE polarization. From the simulated results it can be noted that when the array of the FSS is bent, the resonant frequency is shifted from $10.1 \mathrm{GHz}$ to $9.5 \mathrm{GHz}$ resulting in an alteration of $6 \%$. Furthermore, the bandwidth performance is reduced from $19 \%$ to $15.8 \%$. The bandwidth reduction of $3.2 \%$ occurs when the FSS array is bent at a radius of $150 \mathrm{~mm}$ and the same occurs with a bending radius of $200 \mathrm{~mm}$. The shift in the resonance frequency and the reduction of bandwidth is mainly attributed to the fact that the reflective index of the FSS is varied when it is curved [15]. For the planar FSS, both through and reflected electromagnetic waves form a planar wavefront. However, for the curved FSS, a spherical wavefront is formed as a result of it interaction with the impinging electromagnetic wave, which results in a change of the reflective index of the FSS. Therefore, several bending angles will be evaluated in the future to observe how they affect performance of the curved FSS.

Table I shows a comparison of the performance of recently developed flexible FSSs. When compared to [7], the reduction of the bandwidth of the proposed design is less significant. The bandwidth reduction is less significant mainly due to the fact that when the cross dipoles are embedded into the design proposed in [7], the FSS becomes more capacitive which result in a better frequency selectivity [16]. It is worthwhile to note that the findings on the bending effect on the FSS performance is different in [8] and [14], where the bandwidth is enhanced for the curved structure. The enhancement of the bandwidth is the result of the edge diffraction effects, where multiple ripples were formed, which created an additional resonance. Following from these findings, the edge effect may result in either bandwidth enhancement or bandwidth reduction. Thus, it important to find out mitigating techniques of the edge diffraction effects. In this way the proposed FSS can be designed properly for both planar and curved structures.

\section{CONCLUSION}

We have proposed a design of the MRL (Miniaturized Ring Loop) FSS (Frequency Selective Surface) that is realized by embedding four slots and four cross-dipoles at each of the $90^{\circ}$ into the convetional ring loop FSS. The proposed MRL had been reduced to the dimension of $0.25 \lambda_{\circ}$, where $\lambda_{0}$ is the free-space wavelength at the center frequency.. The

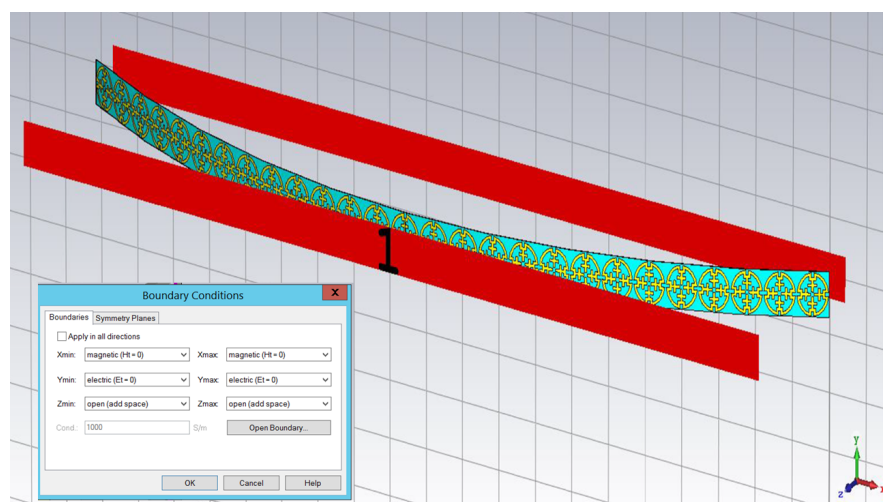

Fig. 3: Simulation setup for the investigation of the conformal MRL FSS array using CST

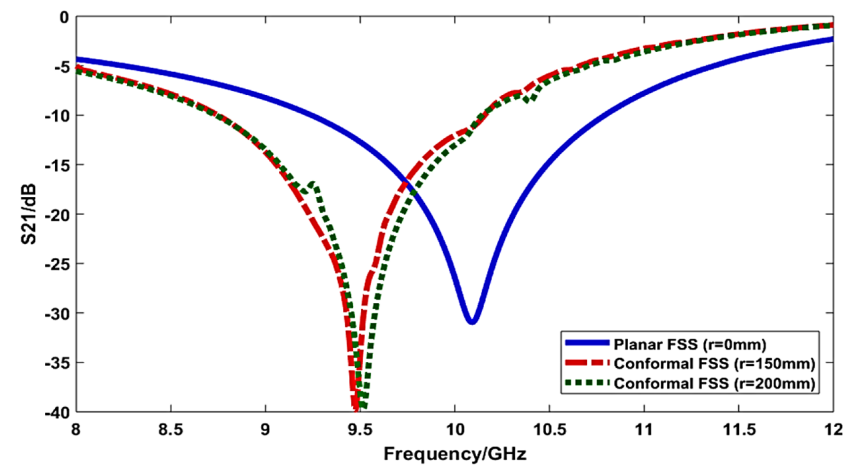

(a)

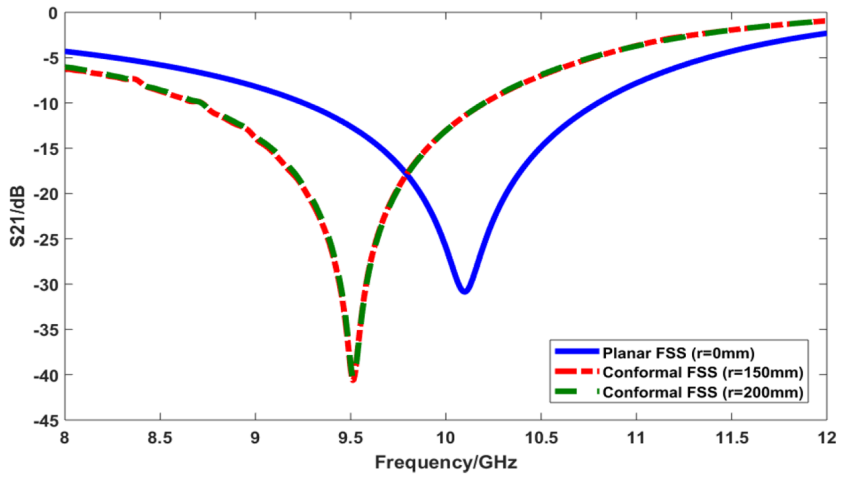

(b)

Fig. 4: Comparison of the transmission response for planar and the conformal MRL FSS array for (a) TE polarization and (b) TM polarization 
proposed structure provides a stable transmission response up to $60^{\circ}$ in the planar feature. We also investigated the impact of bending a MRL FSS linear structure on its frequency response performance by means of the semi-infinite modelling technique. We conclude that the proposed flexible MRL FSS can be operated in both planar and curved surfaces at the $\mathrm{X}$-band as desired. Moreover, as a result of our study we have observed that the resonant frequency (around $10 \mathrm{GHz}$ ) is shifted by $6 \%$, which is not a major deviation. On the other hand, the bandwidth performance of the MRL FSS is reduced by around $3 \%$, which can be explained by ripple effects due to edge diffraction and the change of the reflective index of the curved surface of the MRL FSS. In addition, the semiinfinite modelling technique was shown to be feasible for the evaluating of the bending impact on the performance of the curved FSS. This work has opened up several questions that require further investigation. Future work needs to be done to minimize the impact of the variation of the coupling effects among elements and reducing edge diffraction effects for a curved FSS so that the proposed FSS could provide a stable tranmission response at both planar and curved structure.

\section{REFERENCES}

[1] F. Leferink, "Electromagnetic interference: A radiant future!" in Consumer Electronics-Berlin (ICCE-Berlin), 2015 IEEE 5th International Conference on. IEEE, 2015, pp. 206-207.

[2] T. Hartman, N. Moonen, and F. Leferink, "Evaluation of multichannel synchronous conducted tdemi measurements for high voltage power electronics," in 2018 International Symposium on Electromagnetic Compatibility (EMC EUROPE). IEEE, 2018, pp. 839-843.

[3] B. D. Oakes, L.-G. Mattsson, P. Näsman, and A. A. Glazunov, "A systems-based risk assessment framework for intentional electromagnetic interference (iemi) on critical infrastructures," Risk Analysis, vol. 38 , no. 6 , pp. $1279-1305,2018$

[4] A. A. Glazunov, M. Bäckström, and B. D. Oakes, "Probability distribution function of the electric field strength from a cw iemi source," IEEE Transactions on Electromagnetic Compatibility, vol. 56, no. 6 , pp. 1550-1558, 2014.
[5] W. Yong, S. Rahim, F. Seman, M. Ramli, and N. Remli, "Miniaturisation of ring shape element frequency selective surface for x-band shielding," in Microwave Conference (APMC), 2017 IEEE Asia Pacific. IEEE, 2017, pp. 877-880.

[6] L. B. Wang, K. Y. See, J. W. Zhang, B. Salam, and A. C. W. Lu "Ultrathin and flexible screen-printed metasurfaces for emi shielding applications," IEEE Transactions on Electromagnetic Compatibility, vol. 53, no. 3, pp. 700-705, 2011.

[7] W. Y. Yong, S. K. A. Rahim, M. Himdi, F. C. Seman, D. L. Suong, M. R. Ramli, and H. A. Elmobarak, "Flexible convoluted ring shaped fss for x-band screening application," IEEE Access, vol. 6, pp. 11 657-11 665, 2018.

[8] A. Chatterjee and S. K. Parui, "Frequency-dependent directive radiation of monopole-dielectric resonator antenna using a conformal frequency selective surface," IEEE Transactions on Antennas and Propagation, vol. 65, no. 5, pp. 2233-2239, 2017.

[9] J. Li, Q. Zeng, R. Liu, and T. A. Denidni, "A compact dual-band beamsweeping antenna based on active frequency selective surfaces," IEEE Transactions on Antennas and Propagation, vol. 65, no. 4, pp. 1542 1549, 2017.

[10] J. S. Chang, A. F. Facchetti, and R. Reuss, "A circuits and systems perspective of organic/printed electronics: Review, challenges, and contemporary and emerging design approaches," IEEE Journal on Emerging and Selected Topics in Circuits and Systems, vol. 7, no. 1, pp. 7-26, 2017.

[11] H. A. E. Elobaid, S. K. A. Rahim, M. Himdi, X. Castel, and M. A. Kasgari, "A transparent and flexible polymer-fabric tissue uwb antenna for future wireless networks," IEEE Antennas and Wireless Propagation Letters, vol. 16, pp. 1333-1336, 2017.

[12] M. Ghebrebrhan, F. Aranda, G. Walsh, D. Ziegler, S. Giardini, J. Carlson, B. Kimball, D. Steeves, Z. Xia, S. Yu et al., "Textile frequency selective surface," IEEE Microwave and Wireless Components Letters, vol. 27, no. 11, pp. 989-991, 2017.

[13] (2014) Datasheet:AgIC ink 1000. [Online]. Available: https://agic.cc/en

[14] A. Chatterjee and S. K. Parui, "Beamwidth control of omni-directional antenna using conformal frequency selective surface of different curvatures," IEEE Transactions on Antennas and Propagation, vol. 66, no. 6, pp. 3225-3230, 2018.

[15] R. C. Rumpf, M. Gates, C. Kozikowski, and W. A. Davis, "Guided-mode resonance filter compensated to operate on a curved surface," Progress In Electromagnetics Research C, vol. 40, pp. 93-104, 2013.

[16] T.-W. Li, D. Li, and E.-P. Li, "A novel fss structure with high selectivity and excellent angular stability for 5g communication radome," in 2017 10th Global Symposium on Millimeter-Waves. IEEE, 2017, pp. 50-52. 